\title{
A scientific method for forensic examination of paper
}

\author{
Reeta R Gupta \\ Senior Scientific Officer-cum Assistant Chemical Examiner to the Government of India Central Forensic Science Laboratory \\ (CBI) New Delhi, India
}

*Corresponding Author:

Email: reetacbi@gmail.com

\begin{abstract}
In the modern society, it is surprising to know that criminals are well equipped with novel technological facilities and they have adopted numerous methodological ways for conducting highly organized crime. Due to the immense advancement in Science and Technology, criminals are being quicker in exploiting sophisticated scientific technology for criminal activities and desires to commit crime. Hence the more complex criminal cases are gradually increasing in number and are becoming a major challenge for forensic document experts relating with paper and ink examination, such as paper analysis for differentiating between forged and originality, ascertain authenticity of paper document, its identification, relative age of documents and document dating. The present write-up is an attempt to describe overall approach in forensic examination of paper for distinguishing between two or more sets of paper. Chemical and physical analysis of paper by using appropriate scientific method provides valuable information regarding the details of the document. However application of advanced sophisticated techniques and appropriate scientific methods for reliable results without destruction of the original documents are the prime requirements of the forensic document expert and investigating agencies.
\end{abstract}

Keywords: Document examination, Paper differentiation.

\section{Introduction}

In the modern society, it is surprising to know that criminals are well equipped with novel technological facilities and they have adopted numerous methodological ways for conducting highly organized crime. Due to the immense advancement in science and technology, criminals are being quicker in exploiting sophisticated scientific technology for criminal activities and desires to commit crime. Hence the more complex criminal cases are gradually increasing in number and are becoming a major challenge for forensic document experts relating with paper and ink examination, such as paper analysis for differentiating between forged and originality, ascertain authenticity of paper document, its identification, relative age of documents and document dating. Ink used with writing instruments and paper both are questioned in variety of criminal cases. Queries are often asked for comparison between two or more paper. Enhancement in criminal activities also affect the economic status of our society as well as country, thousand millions of Indian rupees of the Government revenue is put to loss by bank frauds, cheating, forgeries etc. in heinous crime, the documents involved are needed to be scientifically examined in a manners so that the evidential value of documents are not harmed.

Forensic examination of paper involves analysis, comparison and evaluation of questioned and standard document for ascertaining its authenticity. In connection with examination of documents authenticity of paper may be questioned in many cases such as cheating, forgeries, bank fraud, stock fraud, insurance fraud, tax evasion, anonymous letters, wills copyright dispute etc. Paper is one of the most important key used for write on or print on. Chemical and physical analysis of paper by using appropriate scientific method provides valuable information regarding the details of the document. The method of choice for any given situation is determined by several factors, including: the objectives of the investigator e.g. authentication and the relative value of the paper e.g. historically, legally, personally. The word "paper" is derived from the name of the plant papyrus; however the true paper is made of pulped cellulose fibers like wood cotton or flax. Pulp is classified according to pulp production method either chemical or mechanical. ${ }^{1,3}$ Better quality of paper is made by chemical method. Most of good quality of paper is consist largely of chemical good pulp and with a small rag pulp to improve its properties ${ }^{4}$. The best known filler with which paper is loaded is kaolin or china clay, calcium sulphate and barium sulphate. The objective of this paper is to describe various advance methods which is useful for identification and discrimination of paper for forensic purpose in order to solve the criminal cases. In the Forensic laboratory, the query related with paper examination is generally askes as when the paper was first manufactured related with its source of origin. Sometime the query is also asked for physical and chemical erasures on paper. Mechanical erasures will disturb the upper fibers of the paper and attempt of erasures can be visually apparent on the paper surfaces.

Methods for Examination of Paper: Application of advanced sophisticated techniques as well as appropriate scientific methods for quick reliable results without destroying or disfiguring the original documents are the prime requirements of the forensic document expert and investigating agencies. The scientific examination of paper are conducted with various parameters such as color, surface appearance, 
coating, loading materials, brighteners, watermark, weight or thickness, inclusion, by using ultra-violet and microscopic examination methods. During forensic examination of paper other parameters are also taken in consideration including touch, feel, Shake, fold of the sheet, shape, size, opacity, brightness, gloss, smoothness, and burst and tearing strength etc. A mark or image that is not visible under normal light condition which is through naked eye and may be clearly revealed under ultra-violet or Infra-red light conditions. A qualitative analysis gives detail information regarding the constituent materials used in the paper such as fibers, fillers, etc. Chemical analysis evaluates composition and $\mathrm{pH}$; Physical analysis measures gloss, strength, color; Organic analysis detects carbon-based traces of plants and organisms; while inorganic analysis identifies mineral evidence in pigments and dyes.

The forensic document examiners prefer only nondestructive technique for solving the various problems related with forensic document examination. For the purpose of paper examination the chemical and physical process through which the paper is subjected during its manufacturing process provides the detailed identifying characteristic features which allow the questioned document expert to discriminate in between two or more paper samples. The detail characteristics available in standard paper are first examined with help of scientific tool and then its comparison is carried out with the questioned paper document. The identification of genuine paper/distinguishing between two or more sets not an easy task. The difference can be noticed when the suspected document is examined thoroughly under a microscope using various light source arrangement as well as by allowing the oblique light to strike the paper from one side.

Examination conducted under ultra-violet light source, infrared luminescence may also use to study the paper fibers. Paper fibers can be easily seen by microscopic as well as optical method. Ultra-violet and fluorescence microscopes are powerful tool for paper analysis. The main advantage of using ultraviolet light is that it is strongly absorbed by paper material and producing good contrast of image. The ultra-violet microscope is useful for the study of lignin of paper. Generally after visual and optical examination of paper, the forensic document expert takes inference to select an appropriate validated method for further process of examination to conduct the detail scientific analysis of paper components by applying either physical, chemical or spectroscopic techniques depending upon the question asked. The selection of method is best known by the forensic document expert accordingly with the requirement of the examination in order to solve the criminal case. Many at times, when the result is not precise or accurate, the expert has to make decision to use different methods to reach on a conclusion.
Optical Examination of Paper: an optical examination is preferred when the document expert has to use a nondestructive method for examination of document to maintain the originality of the document without destructing it. In paper fibers, most of the microscopic details of wood are destroyed during the pulping, bleaching and refining processes. Thus optical microscopy has always played an important role in paper examination research to understand the morphology of wood in paper making. 5 Optical microscope can be used with incident or transmitted light or a combination of these depending upon the requirement of examination. Practically the preliminary examination of questioned document starts with optical examination. Optical examination involves observation of paper under visible/natural light, ultraviolet light, and filtered light or near infrared regions of electromagnetic spectrum. Visual color matching is still being use for comparison of questioned and standard paper. Human eye is quite sensitive to the differentiation of color of dyes. Using these methods the document expert analyzes the color, luminescence of paper, security fibers and watermarks in paper inserted during the paper manufacturing process. In general practice the range of illuminants is created by different types of lamps using arrangement of different filters in instruments such as Video spectral comparator. ${ }^{6}$ The morphology of paper can easily studied by optical examination of paper. Stereoscopic microscopy allows viewing the paper sample in three dimensional by using incident light with stereomicroscope at low power gives good depth of field. Hence morphological study of surface structure is possible to understand in detail by applying this method. Compound microscope is a classical tool for fiber and pulp analysis of paper. ${ }^{7}$ It is also useful in forensic analysis of fillers and coating particles of the paper. Optical microscopy is very useful for examination of cross sections of fibers within paper sheets.

Watermark Identification: Forensic examination of paper based on its constituent requires two major factors to produce significant result. First preliminary examination of paper carried out for study of its source of origin i.e. manufacturer of paper and second the detailed constituent of paper content. Since watermark is prepared during the time of paper manufacturing process and it is an integral part of the sheet. Hence watermark examined in papers are highly useful in tracing the manufacturer as well as commercial distribution of watermarked paper. ${ }^{8}$ Identification of watermarks provides information regarding manufacturer of the paper which can be important aspect and used as valuable for prove of evidence in establishing the authenticity or spuriousness of documents. This is a common method of determining the source of paper origin, by knowing the watermark manufacturer, the relative age of the paper can be 
determined. ${ }^{9}$ Thus identification of watermark can provide clear indication regarding the degree of the origin of paper whether common or different. The dating of paper by watermark examination is very well known in practice of forensic documents. The specific manufacturer inserts specific individual watermark in the paper during the initial process of paper in the form of code. So coded watermark provide and excellent information regarding the determination/conformation of the date when the paper was manufactured. This is very useful information for paper document dating. Paper containing a specific code in the form watermark if identify, it can linked with paper manufacturer. The yearly published Lockwood-Post's Directory ${ }^{10}$ for example, has a section that associates watermarks with their users. When a paper manufacturer is identified, one first obtains dating information on the watermark to see if this can detect back-dating. The identification as well as differentiation of paper may be determined as following:

1. Identification of coded water mark or the identification of a change in the design of a water mark on a specific date.

2. The comparison of questioned and specimen paper with known standard provided by the manufacturer for the production dates.

3. Elemental composition of the questioned paper obtained from the manufacturer.

4. Presence of trace elemental composition of paper gives very excellent idea about the difference or similarity of papers because the composition of trace element changes if paper manufacture changes the raw materials. The components of paper and their first use in Paper making listed from B.L. Browning ${ }^{11}$ "Analysis of paper" is given in Table 1.

Table 1 shows the introductory date of paper components

\begin{tabular}{|l|l|}
\hline Component & Introductory Date \\
\hline Fibrous Raw Material & 1800 \\
Straw Paper & $1857-1890$ (England) \\
Esparto grass & after.1930 \\
Bleached sulfate wood pulps & $1953-1954$ \\
Organic synthetic fibers & \\
\hline Sizing and Coating Materials & 1937 \\
Soya protein & $1940-1941$ \\
Urea-formaldehyde resin & $1974(1959)$ \\
Dialdehyde starch & \\
\hline Fillers and White pigments & 1920 \\
Barium sulfate & about.1925-1927 \\
Calcium carbonate & after.1932 \\
Zinc Sulfide & about.1938 \\
Di-atomacious earth & \\
\hline Dyes and Colors & 1828 \\
Ultramarine & about 1901 \\
Synthetic organic pigments & about.1950 \\
Optical whitener &
\end{tabular}

\section{Conclusion}

It is remarkable that the forensic document expert interpret examination result based on scientific parameters before the court in any criminal case either ink or paper. Hence experts making result of examination report in the form of opinion with dual objective: first to discover the fact and second to prove the fact in court of law on the basis of observed detailed scientific data. The purpose of forensic examination of paper is to collect all detailed scientific data with available documents by process of comparison and finally to prove before court of law. Document examiners must emphasis the application of advanced optical methods and also by instrumental method for chemical analysis to determine the relative age and source of paper origin. This is useful in compiling chronical data of paper document while giving the answer regarding the relative date of manufacturing document. This can be achieved through systematic and scientific analysis with logical reasoning with reference to the available validate standard data. Thus complete knowledge of instrumentation and application of modern methods/technique will definitely facilitate the forensic document expert to give the meaningful conclusive result to ascertain the authenticity of paper and relative age of document. The research in the ascertaining the age of document is still in its developing stage. The research review is mainly concerned with non-destructive techniques and tools for the comparison, identification of paper. There is a considerable future research requirement in tagging of documents to provide detailed scientific evidence.

\section{References}

1. J Levinsion., Questioned Documents, A Lawyer's Handbook, Academic Press London 2001.

2. Ellen D.M., The Scientific Examination of Questioned Documents, $2^{\text {nd }}$ edn. London; Taylor \& Francis, 1997.

3. Parham R.A. \& Gray R.L., the Practical identification FD wood Pulp Fibers, TAPPI Press.

4. Harision W.R. Suspected Documents Their Scientific Examination, Universal Law Publishing Co. Pvt. Ltd.1996.

5. Rollins, M.L., Cannizzaro, A.M., and Goynes W.R. Light Microscopy in study of cellulose, In Instrumental Analysis of Cotton Cellulose and modified cotton Cellulose. R. T. o' Connor ed, Marcel Dekker, New York, 1972.

6. Mokrzychi G.M., Advancement in Document Examination: The Video Spectral Comparator 2000, Forensic Science Communication, 1999.

7. Iwasaki, T. Lindberg, B. and Meier, H. Paper and Pulp Microscopy. The institute of Paper Chemistry, Appoleton, WI. 1967.

8. Hilton, O., Scientific Examination of Questioned Documents, Elsevier Science Publishing Co. Inc. 1982.

9. Conway James V.P., Evidential Documents, Charles C Tomas USA 1972.

10. Logwood- post's, Directory of Pulp, Paper and Allied Trades, Miller Freman, Inc., San Francisco, C A 1993.

11. Browing B. L., Analysis of Paper, marcel Dekker, Inc. New York 1977. 